\title{
Identidades en contacto en el siglo XVII: la mirada de los italianos sobre la realidad ibérica en las crónicas del viaje de Cosimo III de Medici $(1668-1669)$
}

\author{
Xosé Antonio Neira Cruz ${ }^{1}$
}

Recibido: 01 de diciembre de 2019 / Aceptado: 23 de marzo de 2020

Resumen. Los textos sobre el viaje por España y Portugal realizado por Cosimo de' Medici entre septiembre de 1668 y marzo de 1669, en gran parte todavía inéditos, constituyen una fuente de excepcional interés para profundizar en las relaciones interculturales entre Italia y la Península ibérica. Los autores de los diarios de viaje describen tradiciones y usos culturales locales, la religiosidad y sus manifestaciones, la moda femenina y la gastronomía de los lugares visitados, que ofrecen testimonios aún no considerados por parte de los expertos en estos ámbitos disciplinares. A través de estos testimonios es posible estudiar los contactos lingüísticos con otros pueblos y la diversidad cultural de las regiones que visitaron estos cronistas. Palabras clave: literatura odepórica; Cosimo III de Médicis; tour ibérico; Lorenzo Magalotti; intercultura.

\section{[en] Identities in contact: Italian look over the Iberian reality in the Cosimo III de' Medici's tour chronicles.}

\begin{abstract}
The texts on the trip that Cosimo de Medici made to Spain and Portugal between September 1668 and March 1669, which are mostly unpublished, are a source of great interest to deepen in the intercultural relations between Italy and the Iberian Peninsula. The authors of the travel diaries describe traditions related to local cultures, religion and its manifestations, female fashion and cuisine of the countries that have been visited, which provides testimonies that have not been considered by experts in the field yet. Hence, through these testimonies it is possible to study the linguistic contacts with those countries as well as their cultural diversity.
\end{abstract}

Keywords: Italian travellers in Spain and Portugal; Cosimo III of Medici; identities; intercultural contacts; XVII century.

Sumario: 1. Introducción: un príncipe viajero 2. Usos y costumbres locales: curiosidad desde la superioridad 3. La religiosidad: crítica a las supersticiones locales 4 . La moda: el tour de las damas 5. La gastronomía: el tour de los sabores 6. Observaciones conclusivas.

Cómo citar: Neira Cruz, Xosé Antonio (2020): «Identidades en contacto en el siglo XVII: la mirada de los italianos sobre la realidad ibérica en las crónicas del viaje de Cosimo III de Medici (1668-1669)», Cuadernos de Filología Italiana, 27, pp. 183-198.

1 Universidad de Santiago de Compostela, Departamento de Ciencias de la Comunicación, Avenida de Castelao, s/n, 15782 Santiago de Compostela.

E-mail: neira.cruz@usc.es 


\section{Introducción: un príncipe viajero}

Cosimo III de' Medici (Florencia, 14 de agosto de 1642 - 31 de octubre de 1723), gran duque de Toscana entre 1670 y 1723, sexto y penúltimo de los Medici sobre el trono gran ducal, ha pasado a la historia como uno de los grandes viajeros de su momento, también uno de los monarcas más versados en cuestiones relacionadas con la geografía, la cartografía, la botánica y el conocimiento y colección de contenidos que actualmente calificaríamos como interculturales, virtudes estas que, sin embargo, han quedado empañadas por la negativa valoración histórica de Cosimo III como gobernante.

Cuando todavía era gran príncipe de Toscana, es decir, heredero del trono gran ducal, Cosimo de' Medici acometió una serie de viajes de larga duración que comenzaron en 1667 y se prolongaron, con breves intervalos de estancia en Florencia, hasta el 1 de noviembre de 1669, fecha de su definitivo regreso a la capital toscana. En su último grand tour dedicó unos siete meses a recorrer la Península ibérica, periplo perfectamente documentado en la relación oficial del viaje, así como en las crónicas y diarios personales escritos por sus acompañantes, entre los que destaca la presencia, como autores de dichos textos, del literato Lorenzo Magalotti, del diplomático Filippo Corsini o del médico Giovan Battista Gornia.

En este artículo nos ocuparemos de presentar las impresiones que España y Portugal causaron en los toscanos. Por razones de espacio, delimitaremos nuestra contribución a los siguientes ámbitos temáticos sobre los cuales los cronistas del viaje fijaron especialmente su atención: los usos y costumbres culturales locales; la religiosidad y sus manifestaciones, especialmente aquellas que diferían de las practicadas en Italia; la moda femenina y las gastronomías española y portuguesa, referencias interesantes para advertir la labor comparatista intercultural que las crónicas del viaje representan

\section{Usos y costumbres culturales locales: curiosidad desde la superioridad}

En el sentido amplio de la palabra, cultura lo es prácticamente todo. Desde la literatura y la música hasta las artes plásticas y escénicas, pasando por los usos, costumbres, tradiciones, la moda, la gastronomía o el hábitat, como espacio construido o naturaleza moldeada por la mano del hombre teniendo en cuenta una intencionalidad estética. De todas estas dimensiones culturales podemos encontrar abundantes referencias en los diarios del viaje de Cosimo de' Medici, aunque, por su propia naturaleza, hemos preferido presentar aquí aquellas actividades más propiamente relacionadas con las producciones culturales del momento, dejando para el punto siguiente aquellas en las que la cultura se hibrida de forma inextricable con la práctica religiosa.

El príncipe y su séquito admiran el paisaje ibérico, destacan los constantes contrastes que van advirtiendo y, de hecho, se convierten en unos cronistas fieles de la geografía por la que transitan -no en vano el heredero toscano sintió y cultivó a lo largo de toda su vida una argumentada predilección por esta materia-. Todo esto no les impide elaborar una lista interminable de objeciones ante las muchas aberraciones que encuentran a su paso y que hacen que constantemente su recuerdo vuelva atrás para ponderar la belleza natural de su país de origen. 
Les gustan y atraen nuestros usos y costumbres, más por lo que tienen de exótico y diferente a sus ojos que por su distinción o exquisitez. Algunas prácticas culturales, de hecho, les parecen vulgares o impropias del contexto en el que son desarrolladas, como sucede con los bailes en las vísperas de la Navidad que presencian ante el altar de una iglesia granadina.

Nel dì $18^{\circ}$ detto S.A. si levò a giorno per vedere da una gelosia che guarda in Chiesa lo stravagante costume, che regna tuttavia in molte parti di Spagna e per la festività del Natale nella pubblica Chiesa. Finita la messa cantata comparve a piè de gradini dell'altare un ballerino che aveva più tosto aria di villano vestito d'un drappo bianco, e mavì con calzoni alla Spagnola e camiciola da machera, il quale fatta reverenza all'altare, e tiratosi del corno dell'Evangelio, messo il suo cappello in testa cominciò una lunga ciacona tutta atteggiata di scorci, e mutanze ridicole accompagnate da un concertó di cembalo, chitarra spagnuola, e violino, i quali anche durante la messa, s'intesero spesse volte intramezzati coll'organo. (Sánchez Rivero / Mariutti de Sánchez Rivero 1933: 203)

Demuestran una intención sincera de querer entender, aprender e integrarse como forma de respeto a las culturas con las que están entrando en contacto, no tanto porque les agrade siempre lo nuevo, sino porque forma parte de esa convención o decoro de personas civilizadas y abiertas al descubrimiento y a la sorpresa demostrar dicha actitud. De ahí que no duden en vestirse «a la española» a la primera oportunidad que se les presente, venciendo con redoblada paciencia tanto los hábitos luctuosos que imperaban en la sombría corte de Madrid como la exasperante lentitud de los sastres del país a la hora de tener listo su vestuario. Tanto Magalotti como Corsini se detienen en este tema.

S.A. il dì primo d'Ottobre si messe in abito alla Spagnola, il simile fece il Sig.r Cavalier Dante solamente, non avendo i sarti finiti gl'abiti per alcuno degl'altri Gentiluomini [...] Lunedì $1^{\circ}$ ottobre escì fuori S.A. con tutta con tutta la sua comitiva vestita alla spagnuola nella conformità che usano i soldati, cioè a dire calzone stretto e giustacuore corto e largo, con cravatta e cappello quasi alla francese... (Sánchez Rivero / Mariutti de Sánchez Rivero 1933: 30)

Si bien se advierte una actitud de superioridad cultural con respecto a los países y pueblos que están conociendo, su interés resulta genuino, y no escatiman elogios cuando creen que son merecidos. La música, la arquitectura²,

Magalotti pondera las obras inacabadas de un palacio que considera lo más digno de reseña que encuentran en El Carpio (Córdoba): «Il Carpio è un Villaggio di 380 fuochi, nè altro vi è di considerabile, che un magnifico principio di palazzo che con la morte del padrone rimase poche braccia alto da terra. Il diseño è di Baccio del Bianco ingegner fiorentino, che morì non molti anni sono al servicio di Filippo IV ${ }^{\circ}$. L'architettura è all'italiana e il materiale son due sorte di pietra, rossa per il massiccio, bianca per i conci, l'una e l'altra assai dura». Corsini añade datos para identificar definitivamente este edificio: «...fu questo cominciato da Don Luigi de Aro, con intenzione di fare una gran macchina, con giardini e fontane, ma sopraggiunto dalla morte quasi sul principio restò l'opera imperfetta, et il Marchese di Licce suo figliolo la lascia anch'egli in abbandono...» (Sánchez Rivero / Mariutti de Sánchez Rivero 1933: 174). El referido promotor de la obra fue, efectivamente, Luis Méndez de Haro y Guzmán de Sotomayor (Valladolid, 1598 - Madrid, 1661), VI marqués del Carpio, I duque de Montoro, II marqués de Heliche y II Conde-Duque de Olivares, sobrino y sucesor de Gaspar de Guzmán Pimentel Ribera y 
la escultura ${ }^{3}$-cuando es de precio o cuando evidencia influencia italiana- y las colecciones de pintura, especialmente las atesoradas en los reales sitios del Alcázar de Madrid y San Lorenzo del Escorial, «che è una Fabbrica la più grandiosa, e la più Magnifica che sia in tutta l'Europa» (Sánchez Rivero / Mariutti de Sánchez Rivero 1933: 130), suelen concentrar esa admiración, manifestada de forma muy clara en algunos casos. En otros, el tamiz comparativo hace que lo que se les antoja agradable resulte incompleto por la referencia nacional de la que parten y con la que, de forma casi inevitable, confrontan lo que van conociendo. Así lo manifiestan cuando se acercan a la Casa de Campo de Madrid.

Nel tornare a casa S. A. smontò a veder la casa del Campo, antica abitazione di delizie de' Re di Spagna [...]. Per un portone posto su la strada che va lungo il Manzanares, e che non ha nulla di regio s'entra in un praticello che su la mano manca

Velasco de Tobar (Roma, 1587 - Toro, 1645), el poderoso valido de Felipe IV. Aunque no alcanzó la notoriedad de su tío, Luis de Haro estuvo al frente de importantes empresas políticas no siempre resueltas con acierto. Se ocupó del Tratado de los Pirineos, firmado con Francia en la Isla de los Faisanes en 1659, y consiguió aplacar la insurrección catalana recuperando Barcelona en 1652, pero fracasó estrepitosamente en la guerra contra Portugal, asumiendo personalmente la gran derrota de las tropas españolas en la batalla de Linhas de Elvas (1659). Su hijo, Gaspar Méndez de Haro y Guzmán Fernández de Córdoba (Madrid, 1629 - Nápoles, 1687) es el «Marchese di Licce» al que se refiere Corsini. Fue también él mismo un destacado político y diplomático, embajador en Roma -en donde se enfrentó al papa Inocencio XI en defensa de los derechos y privilegios del Palazzo di Spagna, sede de la cancillería española, y su jurisdicción sobre los barrios romanos adyacentes- $\mathrm{y}$, por mor de las presiones pontificias, promocionado por Carlos II a virrey de Nápoles, en donde murió. Gran mecenas e importantísimo coleccionista de arte, «hombre de fuerte carácter, compleja personalidad y gran cultura» así como «de malas costumbres», el marqués de Heliche ha pasado a la historia del teatro por su importante papel en la corte de Felipe IV como responsable de los festejos reales del Parque del Buen Retiro, en donde trabajó codo con codo con el dramaturgo Pedro Calderón de la Barca (Madrid, 1600 - 1681) y con el escenógrafo Baccio del Bianco (Florencia, 1604 - Madrid, 1657), enviado por el gran duque de Toscana, Ferdinando II de' Medici, a la corte de Madrid, a petición de Felipe IV, para dar realce al programa de espectáculos y fiestas que el rey -apasionado del teatro y de la poesía-impulsó y promocionó. «Liche formó [...] un "equipo artístico" de primer orden que resultó decisivo para la evolución de la fiesta teatral cortesana cantada, la solución hispana a los experimentos que sobre el teatro musical desarrollaron diferentes cortes europeas durante el primer barroco» (cf. Flórez Asensio 2010: 145). Fue, por otro lado, un personaje político muy activo e importante dentro del partido donjuanista.

3 Es el caso el retrato ecuestre de Felipe III que Cosimo III admira en los jardines de la Casa de Campo. «Fra la fontana e la casa [de Campo] v'è sopra un piedistallo assai galante di marmo di Carrara un cavallo di bronzo Donato da un Gran Duca della Serenissima Casa Medici con la statua di Filippo Terzo armato» (cf. Sánchez Rivero / Mariutti de Sánchez Rivero 1933: 89-90), "Il Caval di Giambologna", como puntualiza Corsini (Sánchez Rivero / Mariutti de Sánchez Rivero 1933: 90), obra efectivamente iniciada por del escultor franco-florentino Jean Boulogne (Douai, 1529 - Florencia, 1608), más conocido como Giambologna, y terminada, tras la muerte de este, por su discípulo y continuador, Pietro Tacca, en 1616. Esta escultura, regalo de Cosimo II de' Medici a su cuñado, el rey español, ha vivido una historia verdaderamente azarosa. Recibida inicialmente en la corte con bastante indiferencia, «durante algún tiempo estuvo metida dentro de su caja delante del Alcázar, mientras que la gente de Madrid se arremolinaba a su alrededor queriendo ver por las rendijas del contenedor de qué se trataba aquel regalo» (cf. Salort Pons, 2004: 351), fue ubicada en la Huerta de la Priora, uno de los jardines del Alcázar Real, si bien poco después fue movida a la Casa de Campo, en donde la encuentran Cosimo y su séquito en 1668. En 1848 la reina Isabel II autorizó su traslado a la Plaza Mayor de Madrid, siendo desmontada en 1873 por orden del gobierno de la Primera República. Permaneció guardada en unos almacenes municipales hasta que dos años después fue restituida a su pedestal en la Plaza Mayor. Tras la proclamación de la Segunda República, en 1931 sufre un importante ataque y es derribada, siendo posteriormente restaurada por Juan Cristóbal y recolocada en el centro de la Plaza Mayor, donde sigue actualmente. Resulta, por otra parte, interesante seguir la evolución de las relaciones artísticas entre las cortes madrileña y florentina a través de los retratos ecuestres de monarcas españoles realizados por escultores florentinos y enviados a Madrid como regalo de sucesivos grandes duques toscanos. 
ha una spezie di bettola, in faccia s'inalza verso alcuni monticelli poco deliziosi, e su la man dritta si ristringe in un viale assai corto che conduce alla casa del Re, che in Toscana non sarebbe punto impropria alla condizione d'un commodo cittadino. (Sánchez Rivero / Mariutti de Sánchez Rivero 1933: 89)

Lo mismo sucede cuando, observando la esbelta silueta de la Giralda de Sevilla, se les escapa, casi sin querer, el reproche añadido: «Il campanile è reputato il più sontuoso di Spagna; la struttura rende qualche aria a quello di San Marco di Venezia», comenta Magalotti, «...benchè sia bello non parrebbe tanto in Italia», remacha Corsini (Sánchez Rivero / Mariutti de Sánchez Rivero 1933: 224).

Admirar templos se convierte en uno de los pasatiempos predilectos de Cosimo de' Medici. No se cansa de entrar y salir de ellos tejiendo rutas encadenadas e interminables de capillas, monasterios, oratorios y demás recintos de índole religiosa a los que dedica mañanas o tardes enteras de visita cultural y, por supuesto, religiosa, en las que no falta la oportunidad para los encuentros sociales, diplomáticos o políticos desarrollados al amparo de la discreción de los recintos sagrados. Sirva de ejemplo el periplo desarrollado en Madrid el 29 de octubre de 1668.

S.A. [...] in su le 13 e mezzo ${ }^{4}$ si portò a udir la messa al Carmine. Questa Chiesa è moderna con una sola nave e due ale di cappelle [... Dal Carmine fu S.A. a Nuestra Sennora de la Soledad, Immagine avuta in grandissima venerazione, all'Ospedale degl'Italiani, e a gli Agostini Scalzi detti Agostinos Recoletos tutte piccole Chiesette. In quest'ultima s'entra dalla Chiesa in una Cappella molto abbellita di dorature, dov'è un'Imagine di Nostra Signora, di grande devozione in Madrid [...] Dopo desinare S.A. andò a visitar la Chiesa de Gesuiti [...] La chiesa è una croce assai grande co' pilastri di nuovo ordine per l'impropria mescolanza del dorico e del corintio. La volta è ornata di scorniciamenti di stucchi dipinti a fresco [...] La cupola non è a catino, ma ha il suo tamburo illuminato con spesse finestre: la centinatura però della volta cova infelicemente. Della Chiesa salì S.A. nella libreria ${ }^{5}$ [...] Da i Gesuiti andò a veder la nuova cappella di Sant'Isidoro, congiunta alla parrocchia di Sant'Andrea [...] La Chiesa è rifatta quasi tutta di muro, ma con tutto ciò rimane assai piccola e senza ornamenti [...] Da Sant'Isidoro si portò a San Francesco chiesa ordinaria assai con due altre chiesette o più tosto cappelle annesse una detta de Nuestra Senora de l'Aurora, Immagine di rilievo tenuta con gran venerazione fra lampade di argento, e abbigliamenti sacri ricchi d'oro e di gioie, e l'altra del Santo Cristo, per conservarvisi un Crocifisso di somma devozione. (Sánchez Rivero / Mariutti de Sánchez Rivero 1933: 92-93)

Jornada verdaderamente maratoniana en la que la devoción principesca se da la mano constantemente con la cultura, tanto al ponderar las tradiciones relacionadas con cada lugar como, sobre todo, al considerar la calidad de cada fábrica y las características arquitectónicas o decorativas de los recintos visitados, dimensión esta a la

$4 \quad$ Es decir, a las siete y media de la mañana, según nos aclaran Sánchez Rivero y Mariutti de Sánchez Rivero (1933: 91).

«...ben ornata di quadri con i ritratti di Santi et huomini illustri dela lor Compagnia» (Sánchez Rivero / Mariutti de Sánchez Rivero 1933: 92), como añade Corsini, deteniéndose en una sucinta descripción de los contenidos de dicha biblioteca . 
que tanto Magalotti como Corsini y Gornia consagran páginas enteras de sus diarios. Sin embargo, como sucede por otra parte a lo largo de todo el viaje y no solo en lo relativo al arte, a veces, incomprensiblemente, se quedan fuera de las apreciaciones de los cronistas -y suponemos que de las visitas realizadas por el príncipe de Medici y sus acompañantes- obras clave del patrimonio español o portugués.

[...] curioso resulta también el hecho de que Corsini, que suele ser bastante puntilloso en la descripción de los ceremoniales, patrimonio artístico y creencias populares, no mencione - dentro del aspecto urbanístico de Compostela- una joya arquitectónica tan importante como el Pórtico de la Gloria, al igual que los otros relatores. (González Fernández 2004: 247)

En este sentido, hay que tener en cuenta que ni los florentinos demuestran especial aprecio por el románico o el gótico como estilos que llamen su atención ni el Pórtico vivía sus mejores horas en cuanto a la estima general.

No encontramos referencias demasiado explícitas ni admirativas a las literaturas escritas en las lenguas peninsulares a lo largo de las páginas de los diarios, quizás porque no tenían buena opinión de ellas, como evidencia Magalotti -literato de gran consideración en la Florencia de su momento- en una carta que envía al tío de Cosimo, el cardenal Leopoldo de' Medici, el 16 de noviembre de 1668 desde Madrid: «Toda la presente literatura de España se reduce a teología escolástica» (Sánchez Rivero / Mariutti de Sánchez Rivero 1933: xxi). Tan severa y desproporcionada aseveración nos deja ver un flanco por el cual la documentación sobre España y Portugal que los florentinos manejan -tan detallada, incluso, a veces, prolija- flaquea. Se escapa a tan injusto juicio un género que vive una época de oro en España: el teatro. Sobre este tema nos remitimos a Neira Cruz (2016).

\section{La religiosidad: crítica a las supersticiones locales}

Resultaría interminable hacer el recuento de las ocupaciones de Cosimo de' Medici relacionadas con la práctica de devociones religiosas, pues no hay ni un solo día a lo largo del viaje en el que no escuche misa, rece, visite iglesias o conventos, rinda culto a imágenes y reliquias o mantenga encuentros con personas relacionadas, de un modo u otro, con la Iglesia. Esa preferencia -que configuró decisivamente no solo la personalidad y gobierno del penúltimo gran duque mediceo de Toscana, sino también la impronta que de él ha quedado para la posteridad-determina su deseo de acercarse a los principales santuarios que figuran en su ruta o a los que se desplaza expresamente para participar en liturgias y ceremonias presentadas en los diarios con todo lujo de detalles. Montserrat y el Pilar son, en ese sentido, paradas obligadas para el gran príncipe de Toscana, de las cuales, como se puede apreciar, no salió defraudado. Toda la riqueza, complejidad y solemnidad de los actos de carácter litúrgico y devocional desarrollados adquieren, en estos fragmentos debidos a Magalotti, su máxima expresión.

Arrivata l'A.S. alla Chiesa [de Montserrat] fu ricevuto fuori di essa alla porta del convento col suono delle campane dall'Abate con alcuni Monaci e con molti Chierici con cotte, sonando tutti diversi strumenti di fiato. Avvicinatosi alla porta 
della Chiesa vecchia, ridotta presentemente a un passaggio per andare alla nuova, trovò sopra di essa un Monaco in piviale in mezzo e due Chierici con candellieri d'argento, a pie de' quali vi era un cuscino di velluto rosso, sopra il quale S.A. inginocchiatosi baciò un Crocifisso d'oro smaltato portoli dal suddetto monaco. Levatosi poi e postosi poi secondo il cerimoniale prescrittogli dai Monaci tra il $\mathrm{XP}^{\mathrm{o}}$ e l'abbate s'intonò il Tedeum, e camminando processionalmente dietro un'altra croce tutti i Monaci et in mezzo la musica si passò un cortiletto che rimane tra le due Chiese, e s'entrò nella nuova. Giunti all'Altar maggiore il Monaco portante il Cristo salì i gradini di esso e posato il Cristo su l'altare tiratosi dal corno dell'epistola intonò il "Salvum fac servum tuum Domine" con altre orazioni sopra la persona di S.A. che stava inginocchiato su lo strado a pie dell'altare del Corno dell'Evangelo. Finite l'orazioni il Monaco ripreso il Cristo si ritiò in Sagrestia, e allora i Cantori saliti più vicini all'altare intonaron la Salve dopo la quale udita da S.A. la messa accompagnato dall'Abate e da alcuni monaci alle sue stanze, ivi desinò, regalato dal Convento di frutte, d'ulive e di vino [...]. Finita la messa, durante la quale i cantori nella camera dietro la Cappella cantarono diversi mottetti, s'aperse la porta che risponde dietro l'Immagine di Nostra Signora, e chiuso il velo che la copre alla Chiesa, S.A. Le baciò la mano. (Sánchez Rivero / Mariutti de Sánchez Rivero 1933: 40-41)

$[\ldots]$

Dal passeggio S.A. si roportò a Nuestra Senora del Pilar Chiesa assai grande e antica col coro in fondo e che nell'architettura non ha niente di singolare. Quivi S.A. scese nella Cappelletta che si dice fabbricata per mano di S. Giacomo Apostolo con l'aiuto degl'angeli. (Sánchez Rivero / Mariutti de Sánchez Rivero 1933: 58)

Cuando el príncipe recibe noticia de la existencia de alguna devoción local desconocida para él - como es el caso del Santo Corporal de Daroca- se apresura, como no podía ser menos en él, a participar en ella. «Riserrata la custodia d'oro (che dicono esser fatta col prim'oro che venisse dall'Indie) e tornata a coprir col drappo il Sacerdote la pose in su la testa di S.A. et in riguardo suo, per special grazia sopra quella di tutta la sua comitiva» (Sánchez Rivero / Mariutti de Sánchez Rivero 1933: 71). Y desde luego, no renuncia a conocer de cerca demostraciones de la tradición religiosa que le llaman la atención por su exotismo o excepcionalidad, como sucede en la catedral de Toledo con la misa por el rito mozárabe: «Nel dì 28 detto intorno alle 8 ore della mattina S.A. uscì in carroza, e andò alla Cattedrale, dove era aspettata nella Cappella fabbricata dal Cardinal Ximenes per entender quivi l'ore, e la messa secondo il rito Mosarabe» (Sánchez Rivero / Mariutti de Sánchez Rivero 1933: 149). Lo mismo sucede en la de Santiago de Compostela con el botafumeiro, cuyo mecanismo, perfectamente descrito por Magalotti, quizás no estuvieran en la época -por lo que se aprecia - tan perfeccionado como en la actualidad o, en realidad, «el botafumeiro de entonces debía de ser de forma más redonda que el actual -realizado por José Losada en 1851-, y quizás más abierto» (García Iglesias, 2004: 593).

Vi è un rito antichissimo d'incensare nelle funzioni, e processioni solenni: Ciò si fa con un incensiere di figura sferica dove la padelletta del fuoco sta messa in bilico. Tutto questo è appeso ad un'ordigno di ferro, che sta dentro la cupola, e volendosi incensare se gli da il moto per via d'una fune, che si ravvolge ad un rocchetto, alla quale essendo diversi capi s'attaccano più persone come al martino, con cui 
si ficcano i pali in terra. Ora cominciando in tal modo l'incensiere a muoversi à guisa di pendolo a poco a poco, se gli va crescendo talmente il moto, che arriva ne i bracci della croce poco meno che a toccar la volta, onde per la furia del moto il fuoco si leva in fiamma, e sfavilla in grandissima copia fuori dell'incensiere. (Sánchez Rivero / Mariutti de Sánchez Rivero 1933: 336-337)

Mientras el príncipe sigue con puntualidad las fiestas del calendario litúrgico, los cronistas no dejan de anotar aquello que, a sus ojos, resulta chocante o diferente, como es el caso de las costumbres asociadas al día de difuntos en la iglesia del Carmen de Madrid, quizás por evidenciar «cierto carácter gentílico» (Sánchez Rivero / Mariutti de Sánchez Rivero 1933: xx). Filippo Corsini nos narra ese momento así:

Il giorno si portò S.A. al Carmine dove in un balcone serrato da gelosie sentí il Vespro cantato in musica da frati, che tra di loro formano un concerto assai buono, quivi si vidde che le donne, e tra esse tal una ve n'era di condizione, costumano di stendere uno strato nero sopra quel sito dove sono sepelliti i loro più prossimi assegnandosi a ciascuna familia in vece di sepolture alla nostra usanza, che tra di loro non si usano, tante braccia di terreno il quale alzando fanno una buca dove posto il cadavere con l'istessa terra cavata lo ricoprono e quella battono e calcano poscia in maniera che ritorni spianata com'era prima et ha questa tal qualità che in termine di 24 ore riducono fin l'ossa in minutissima polvere. Sopra detto strato accendono molti lumi e tal'una vi fa sopra profumi et altre vi pongono sopra alcune ceste coperte, entro le quali ci dissero esservi del pane et altre cose commestibili; e, quivi si trattengono coperte con Drappi Neri finchè si dica l'offizio dei morti. (Sánchez Rivero / Mariutti de Sánchez Rivero 1933: 98)

La sorpresa da paso con frecuencia a la mirada crítica sobre prácticas religiosas o rituales que consideran extraños, inapropiados o directamente grotescos, como señalan al contemplar la tradición del abrazo al Apóstol en Compostela, la cual es considerada ridícula por Magalotti y por Corsini, mientras Gornia se limita a describirla sin emitir juicio al respecto, aunque se aprecie, a nuestro entender, una suave ironía en su forma de contar cómo los peregrinos, al abrazar la imagen del Apóstol, le calzan «il cappello in testa» (Ms. Gornia 1669: 69). Igualmente, al ver la forma en que se confiesan las mujeres en una iglesia de Madrid, sin uso de confesonario y con una excesiva cercanía corporal entre la fiel y el sacerdote, Gornia no deja de asomar su finísima y discreta perspicacia: "Qui si vedde confessare le donne sopra un tappeto a faccia a faccia del confessore, ed alcune alzano il proprio manto, e coprono sotto quello il capo del confessore. Altre coperte servono d'appoggio al confessore, che sulla spalla della penitente posa gentilmente il capo» (Ms. Gornia 1669: 15 v).

Contrastes que enriquecen todavía más el diálogo entre culturas que representan estos documentos y que ofrecen a los expertos o interesados en el desarrollo del ceremonial litúrgico ibérico del siglo XVII, testimonios y descripciones de enorme interés. 


\section{La moda: el tour de las damas}

La historia de la indumentaria peninsular tiene en las crónicas del periplo mediceo una fuente de primera dimensión, no solo por la atención que los acompañantes del príncipe prestan a la forma de vestir y peinarse que practican los españoles y portugueses del siglo XVII -chocante en bastantes aspectos para los florentinos, y achacables, según ellos, a la influencia árabe en la cultura local-, sino por la capacidad gráfica, casi pictórica, de narradores a la hora de describir y plasmar atuendos y combinaciones cromáticas. Sería muy interesante contrastar estos testimonios narrativos con cuadros de la época, a fin de demostrar hasta qué punto texto e iconografía llegan a complementarse a la perfección. Es, en todo caso, una dimensión que no corresponde abordar ahora, limitándonos a traer a colación algunos ejemplos en este sentido, todos ellos premeditadamente extraídos del diario de Gian Battista Gornia. No significa esto que las obras de Magalotti y Corsini carezcan de contenidos igualmente destacables, pero es un hecho que el texto del médico de Cosimo de' Medici alcanza una vivacidad inusitada a la hora de plasmar, con todo lujo de detalles, los usos indumentarios que llaman su atención en España y Portugal, llegando a establecer comparativas entre las distintas regiones por las que transcurre el viaje y aportando datos relevantes sobre la existencia de diversas identidades culturales dentro de un mismo contexto nacional.

Este mérito como observador de Gornia debe conectarse tanto con su curiosidad científica como con su evidente atracción por lo femenino. Que Gornia era un rendido admirador de las mujeres es un hecho constatable al cual, por otra parte, debemos agradecer páginas deliciosas de su relato absolutamente conectadas con la categoría que estamos tratando. He aquí, pues, un tour des dames -si se nos permite la expresión- debida a su pluma, que se inicia con la descripción de la forma de peinarse y rematar sus tocados de las barcelonesas.

Le donne di Barcellona sono bizzarre e anco belle. Queste portano i capelli involti in tre treccie due dalle parti e una di dietro, qle p il più è avuiluppata acutamente in modo che coperta poi con velo ò tela bianca fa una cuffia simile al corno del Doge di Venezia. Molte lasciano queste trecce lunghe qnto sono distese e abbellite con nastri. Scoprono l'orecchie e col busto coprono il seno fino alla gola ma scoprono ${ }^{6}$ le spalle oltre ogni misura. Molte coprono il viso con un velo lungo e cappuccio simile a frati e la maggior parte di esse in età si veste da monaca e di color nero o bigio. (Gornia 1669: $5 \mathrm{v}$ )

Si algunas catalanas entradas en años, como señala el autor, en realidad probablemente viudas, optaban por vestimentas monjiles - muy en boga en un país cuya reina, tras haber enviudado, había adoptaba el hábito religioso como expresión de luto7-,

\footnotetext{
He aquí un ejemplo interesante de contradicción entre el texto original de Gornia y la transcripción de Sánchez Rivero y Mariutti de Sánchez Rivero. Mientras el autor dice «Scoprono», los transcriptores indican «coprono» (37), resultando una descripción del atuendo en cuestión totalmente opuesto.

7 En realidad, la utilización del color negro en España como expresión de luto arranca de la Pragmática de Luto y Cera dictada por los Reyes Católicos en 1502, tras la muerte en 1497 del príncipe Juan, heredero de las coronas de Castilla y Aragón, y de los sucesivos fallecimientos de los siguientes llamados a la sucesión: Isabel, princesa de Asturias y reina consorte de Portugal (fallecida en 1498), y del hijo de esta, Miguel de la Paz, príncipe de Asturias y príncipe heredero de Portugal (muerto en 1500). La necesidad de controlar las manifestaciones pú-
} 
las monjas del monasterio de Santa Lucía, en Zaragoza, llamaron la atención de Gornia justamente por utilizar un atuendo poco acorde con su condición: «Le monache sono licenziosissime e vestite quasi da secolare col guardinfante, capelli lunghi $\mathrm{e}^{8}$ un poco di velo sul capo» (Gornia 1669: $10 \mathrm{v}$ ) . Las seglares no parecen ir a la zaga en la exhibición de su físico, cosa que, como no podía ser menos, no deja de notar nuestro curioso cronista: «le Donne Aragonese ${ }^{9}$ portano d'avanti aperto il busto e parimente la camicia aperta all'usanza degl'uomini ${ }^{10}$ e in vece ${ }^{11}$ di velo in capo si servono di un panno in forma di ferraioletto» (Gornia 1669: $8 \mathrm{v}$ ). No cambia, en todo caso, la forma de vestir de las ancianas o de las viudas zaragozanas «coperte col manto, e solo aperto il viso dalla parte d'un occhio» (Gornia 1669: $9 \mathrm{v}$ ).

A las cordobesas y a las lisboetas dedica también palabras de admiración, especialmente en lo tocante a sus llamativos atuendos. Así, de las primeras dice que «le Dame qui si stellettano di bianco e rosso con gonfi grandissimi alle maniche e pendenti che paiono piramidi» (Gornia 1669: $35 \mathrm{v}$ ), mientras que de las de Lisboa destaca sus tocados: "Qui le Donne portano il suo ferraiolo con un cappello quasi tutto piano grandissimo e con alcuni fiocchi si intorno al capo come nell'estremità della falda sopra la fronte e quelle che non portano cappello vanno tappate con un manto di saiglia nera, che gli copre tutto il capo» (Gornia 1669: $59 \mathrm{v}$ ).

Completa esta atenta aproximación del médico de Cosimo al mundo femenino una encendida alabanza a las capacidades y destrezas de ellas, al afirmar que lo que

blica de duelo y luto - que ocasionaban gastos cuantiosos a las arcas del reino o de las villas y ciudades y que alcanzaban dimensiones desmedidas que iban en contra, a veces, del recato y el recogimiento- llevó a regular la duración de los lutos debidos y los familiares implicados en los mismos en función del grado de consanguinidad existente con el difunto, el gasto en cera aplicable y la utilización del negro (hasta entonces había sido el blanco el color para expresar el duelo) como color que representaba la noche, el miedo y lo tétrico asociado a la muerte (Casas Gaspar 1947). En el siglo XVII las viudas debían amedrentar con sus atuendos, que solían consistir en «negra toca, negro vestido, negra la batista que caía más debajo de las rodillas, negra la muselina que circundaba el rostro y le cubría la garganta, ocultando la cabellera; negro el manto de tafetán que hasta los pies le tapaba; negro el sombrero de anchas alas, sujeto a la barbilla con cintas de seda negra» (Casas Gaspar 1947: 126). Compartían esos lutos los miembros del servicio de las casas principales, los cortinajes y las paredes de las viviendas e incluso las guarniciones de los caballos y los carruajes. En muchos casos, como expresión añadida de celo religioso o de renuncia simbólica a la vida y al mundo tras el fallecimiento del marido, las viudas adoptaban vestimentas muy semejantes a los hábitos de monja, siguiendo los preceptos de guías morales muy leídos en el momento como el Padre Gaspar de Astete (1597: 126) (Coca de Alba, Salamanca, 1537 - Burgos, 1601), quien prescribía que «La mujer viuda debe olvidar su vida matrimonial y entregarse en soledad a la castidad, al ayuno, a la oración y a la limosna: en el momento de enviudar pasa a ser esposa de Dios. El hábito negro ayuda a mantener la castidad y las costumbres ejemplares [...] el vestido exterior muestre el ánima interior [...] la viuda debe dejar todos los trajes que llevaba siendo casada porque un hábito quiere el hombre que traiga su mujer y otro quiere y de otra calidad que traiga la esposa de Jesucristo». Fue el caso de la reina Mariana de Austria, mujer, por otra parte, muy determinada por una devoción religiosa rayana en el fanatismo, quien, a partir del fallecimiento de Felipe IV, vistió un hábito en nada diferente al de una monja, salvo en la calidad de las telas. Se puede comprobar esto en los retratos de la reina regente debidos al pintor Juan Carreño de Miranda (Avilés, 1614 - Madrid, 1685) conservados en las colecciones del Museo Nacional del Prado, del Museo de Historia del Arte de Viena, de la Real Academia de Bellas Artes de San Fernando o del Museo de Bellas Artes de Bilbao, en los que también se advertirá la repercusión del luto en los interiores de palacio; pero también leyendo lo que Gornia dejó escrito tras verla en la audiencia concedida a Cosimo el 7 de noviembre de 1668, podremos corroborar lo señalado: «La Regina è donna grande e di maestosa presenza e vestita da monaca con sei delle sue dame pure vestite da monaca di bianco e nero» (Gornia 1669: 16 v.).

Transcrito sin esta conjunción en Sánchez Rivero y Mariutti de Sánchez Rivero (1933: 63).

Trancrito como «donne Aragonesi» (Sánchez Rivero / Mariutti de Sánchez Rivero 1933: 65).

Transcrito como «degli uomini».

Transcrito como «invece». 
le parece más digno de consideración es el hecho de que «le donne in tutti i negozzi attendono con grandissimo guidizio, et applicazione in modo, che in tutti i mestieri le maggiori fatiche son le loro» (Gornia 1669: $30 \mathrm{v}$ ).

\section{La gastronomía: el tour de los sabores}

Otro de los grandes intereses socioculturales demostrados por los florentinos fue el relacionado con el disfrute de la buena mesa a la manera española. En lugar de trazar una ruta alternativa a la interior -el tour de los sabores, en este caso-, lo cual, nuevamente, exigiría un capítulo completo, por no decir una monografía, dada la cantidad de referencias existentes en los diarios, optamos por recurrir a los trabajos de investigadores que nos han precedido en la atención a este tema con sobrada solvencia. Todos ellos parten de una realidad innegable: Cosimo de' Medici se permitía solo un pecado, el de la gula, el cual tenía una traducción directa en un sobrepeso considerable. Ciertamente, nunca llegó a los límites enfermizos de su hermano el cardenal Francesco Maria de' Medici, pero los comentarios recogidos sobre su físico coinciden en resaltar una corpulencia tendente a la obesidad y, aunque los retratistas de cámara solían ser muy complacientes con sus mecenas, Suttermans ${ }^{12}$, Palloni, Maratta o Cooper confirmaron a través de sus obras esa característica física.

La miniatura realizada en acuarela sobre pergamino por Samuel Cooper (Londres, 1608-1672), que se conserva en la Galleria degli Uffizi ${ }^{13}$ procedente de la colección del cardenal Leopoldo, tío de Cosimo, ha sido datada entre mayo de 1669 y diciembre de 1670, es decir, es el retrato del gran príncipe de Toscana más próximo a las fechas de su viaje por España y Portugal. A pesar del esfuerzo del pintor por presentar a un hombre joven en la plenitud de la virilidad, altivo y de mirada desafiante, no pasan desapercibidos los párpados adiposos, las mejillas rellenas, la prominente papada y una anchura abdominal que ni siquiera la coraza con la que posa consigue disimular. Incluso en una época en la que tener ciertos kilos de más no estaba nada mal visto, sus contemporáneos señalaban «su tendencia a "alimentarse en exceso", y los diplomáticos [...] coinciden al hablar de su "afición melancólica", su precoz "obesidad", y una "intemperancia en la comida" que surge quizás para compensar la extraña abstinencia en la alcoba» (Marchisio 2004: 290). Fue una debilidad que mantuvo a lo largo de toda su vida y que solo llegó a controlar en los últimos años, cuando el iluminado en el que acabó convirtiéndose dejó de necesitar otro alimento que el espiritual.

Los viajes, además de abrir el apetito, sirven para degustar nuevas exquisiteces, de ahí que seguir los pasos del príncipe sea como trazar «una pequeña géographie gourmande» (Marchisio 2004: 290). Junto a él, viajan otros destacados gastrónomos, como es el caso de Magalotti, que había escrito en verso un buen número de

12 El pintor flamenco Giusto Suttermans (Amberes, 1597 - Florencia, 1681), también conocido como Justus Suttermans, admite como variante de su apellido la forma Sustermans, utilizada fundamentalmente en el contexto anglosajón. En Italia se prefiere la forma Suttermans, como lo evidencia la máxima experta actual en este autor, Lisa Goldenberg Stopatto, autora de una tesis doctoral dedicada a este pintor y de una obra más recientemente publicada que lleva por título Un granduca e il suo ritrattista. Cosimo III de 'Medici e la "stanza de' quadri" di Giusto Suttermans (Livorno:,Sillabe, 2006).

13 Inv. $1890, \mathrm{n}^{\circ} 3018$. 
recetas -desde aquella sobre el candiero, que era una especie de helado, hasta la de la mantequilla de almendra y la tortilla a la flor de naranja- y había sido proclamado «patriarca de los sátrapas y los glotones» (Marchisio 2004: 290).

La ruta de los sabores que la famiglia -como le gustaba a Gornia denominar a la comitiva- dibuja por la Península ibérica sirve no solo para saber lo que comieron en cada momento sino para establecer una especie de catálogo o clasificación de las especialidades culinarias más dignas de degustar en cada localidad. Pueblo a pueblo, las frutas de estación van ocupando su lugar sobre la mesa principesca.

«las peras y las manzanas de invierno», «las bergamotas y las ciruelas rojas» de Daroca. Pero en especial las peras y manzanas, a Corsini le habían parecido incluso «milagrosas, con mucho más olor y sabor» que las de Italia. Y no era poco reconocimiento para quien sabía de la increíble variedad pomológica de los Médicis, donde, según la «Lista di tutte le frutte, che giorno per giorno dentro all'Anno sono poste alla mensa dell'Ar. e del Ser.mo Gran Duca di Toscana», crecían doscientos treinta tipos diferentes de peras, que era la fruta más apreciada en aquellos años. (Marchisio 2004: 290)

Hablando de frutas, y de comida en general, Andalucía se lleva los mejores elogios de los florentinos, a los cuales imaginamos

relamiéndose ante [...] las aceitunas aliñadas de Córdoba, los melones amarillos de Écija [...] el jamón serrano de Rute, que nada tenía que ver con los poco curados «prosciutti» italianos de Parma y cuyo secreto radicaba en el sabio uso de la sal, las golosas confituras de las pastelerías granadinas, elaboradas con el blanquísimo y refinado azúcar de Almuñécar y Motril, o el exquisito chocolate que le sirven los canónigos sevillanos. (Palomero Páramo 2004: 483)

El chocolate, ciertamente, merece un tratamiento aparte en ese recuento de manjares, a semejanza de lo que hace Gornia en su diario, en el que dedica una página completa a hablar del origen y propiedades de esta delicatesse para la época, ofreciendo la receta detallada de su preparación y, en definitiva, dejándonos en el paladar el regusto delicioso que el propio médico de Cosimo de' Medici pudo haber disfrutado hace más de tres siglos: «Il cacao è un seme bellissimo simile a pistacchi, alquanto più grosso, e più schiacciato, quale dentro hà la polpa simile di colore e sapore alle nocciole salvatiche ma è di sostanza molto oleosa e pingue in modo che pesto fa subito come un unguento quasi liquido» (Gornia 1669: $14 \mathrm{v} / \mathrm{r}$ ).

No solo para disfrute sino también para sanación del cuerpo se revela adecuado el chocolate a través de las páginas del galeno mediceo, hasta el punto de quedar constancia de que «supo por su colega español, el doctor Alva, que se trataba de un excelente remedio contra la "debilidad de estómago" y como tal había sido prescrito a Felipe IV, pero era nocivo para el hígado y quizás pudiese haber provocado los cálculos renales que, después de la cura, habían afectado al rey de España» (Marchisio 2004: 293).

Lo cierto es que el chocolate resultó una utilísima excusa para trabar complicidades a lo largo del viaje. Así parece, al menos, de lo que se desprende al respecto de la relación oficial de Magalotti, quien, a través del doctor Farina, al servicio del poderosísimo y extravagante cardenal Moncada, duque de Montalto, tejerá un curioso 
cambalache de golosinas entre el prelado y el príncipe. Teniendo en cuenta el papel fundamental del cardenal en el complot político contra la reina regente Mariana de Austria, que se estaba fraguando en las fechas del viaje, apuntamos la posibilidad sustentada en investigaciones anteriores nuestras sobre los contactos políticos de los toscanos en la corte madrileña (Neira Cruz 2015) - de que si no Cosimo, sí miembros de su comitiva pudiesen haber aprovechado dicho juego de recados en torno al cacao y sus múltiples y deliciosas combinatorias, para hacerse llegar mensajes de índole no tan gastronómica. Hablar en clave, en tiempos políticamente revueltos, con una insurrección contra la reina fraguándose al fuego lento del poder alternativo que Montalto, Liche, Carpio y tantos otros estaban conformando en torno a Juan José de Austria, hijo bastardo de Felipe IV, formaba parte de este juego de tronos que se vivió en España en la segunda mitad del siglo XVII, andando, especialmente, los Medici de por medio. En todo caso, es muy probable que el gran príncipe de Toscana permaneciese ajeno a buena parte de la información que sus diplomáticos estuviesen canalizando, hacia Florencia o hacia otras cortes, mientras él se afanaba en cultivar sus «pasiones olfativas y gastronómicas», pues ha quedado buena constancia de «su manía de coleccionar recetas» y el «suspicaz orgullo con el que custodiaba los descubrimientos de sus especieros» (Marchisio 2004: 294).

Si los florentinos no fueron moderaros en el comer, tampoco debieron ser particularmente sobrios con la bebida, pues igual que lo hemos hecho con otros intereses, podría trazarse, siguiendo los textos, una verdadera ruta enoturística de la época.

Entre los vinos aprecian un moscatel blanco de Mora, hasta el punto de acumular una «buena provisión para unos días de viaje». Se elogian los tintos de Consuegra, el andaluz de Monturque, «blanco como agua de color, y de sabor suavísimo» y el gallego, también «blanco y delicado», bastante más apreciado que el vino añejo que probaron en la Cartuja de Granada, «de treinta años tan espiritoso que parecía aguardiente». Cosme bajó en persona a la bodega de la Cartuja, donde el vino envejecía no en cubas de madera, sino en «vasijas grandísimas de terracota». (Marchisio 2004: 290)

En la larga lista de regalos que el príncipe fue recibiendo durante su estancia en España y Portugal, los comestibles ocupan, probablemente, el primer puesto, quizás conocedores sus anfitriones de la debilidad de Cosimo por el buen yantar. Por otra parte, se advierte en varios momentos a lo largo de los documentos, cómo el príncipe o su séquito suelen rehusar aceptar otro tipo de obsequios, llegando incluso a devolver el agasajo acompañado de una gratificación, como sucedió en Córdoba ante la insistencia del caballero local Francisco Los Ríos en regalarle un caballo que al príncipe le había gustado el día anterior. En cambio, sí eran bien recibidos los cestos, cajas, barriles o damajuanas repletos de delicias.

De esta manera, en las antecámaras de Cosme, a menudo a la hora de cenar se recibían regalos y se recompensaba a los donantes con buenas propinas: «una gran jofaina de lenguados y una caja llena hasta la mitad de vino generoso y el resto de vino hipocrático», enviado por el señor Bartolomeo Moneglia de Córdoba, a través de su representante Giovanni Andrea Spinola; «ocho baúles con distintas conservas y confituras y otros con jamones y perdices, y una garrafa de vino» del señor Squarciafico de Granada; un primer regalo «de capones, vino, aceitu- 
nas, manzanas», seguido de un segundo consistente en «una jofaina de lenguados, tres pequeños esturiones, y bastantes melones de Ivera» del señor Giustiniani de Sevilla; sin olvidar «los dieciocho, o veinte recipientes de confituras, y caza» de Lorenzo Bresciani de Elvas; así como algunas «coliflores, muy apreciadas por su rareza» del padre Ardizon de Lisboa. (Marchisio 2004: 291)

No diferían demasiado los presentes llegados de parte de las autoridades. Chocolate y un juego de plata para servirlo y prepararlo fue el regalo enviado a Cosimo por el rey-niño Carlos II de España, mientras el arzobispo de Santiago de Compostela, Ambrosio de Spínola y Guzmán ${ }^{14}$-también él de origen italiano-, sabiendo seguramente de la grata impresión que había causado a los florentinos el pescado gallego («Si pesca nei suoi mari una quantità di pesce et ostriche, che serve per provvedere molti altri luoghi», llega a afirmar Magalotti en su relación; cf. Sánchez Rivero / Mariutti de Sánchez Rivero 1933: 337), correspondió a los ilustres visitantes enviándoles a Coruña algo muy propio del país: una mariscada a base de ostras, varios toneles repletos de lenguados, cuarenta y ocho jamones, frutas en almíbar, jaleas, membrillos..., hasta el punto de sorprender a Corsini semejante abundancia y generosidad (Corsini 1669: 29 v). No es de extrañar que, al hacer recuento de sus experiencias, años después de haber regresado a Florencia, Magalotti se deshaga en alabanzas hacia los españoles considerándolos «la gente más fina y espléndida al regalar que hay en el mundo» (Marchisio 2004: 295).

\section{Observaciones conclusivas}

Concluimos ofreciendo los siguientes puntos de reflexión:

a. Los documentos señalados, que componen el corpus magnum de los textos generados por los acompañantes de Cosimo de' Medici, según la última y renovada clasificación de las fuentes (Neira Cruz 2018), así como buena parte de los manuscritos que conforman el corpus minor, ofrecen un testimonio de primera magnitud para entender un año de la historia de la Península ibérica, contado desde un punto de vista comparatista por parte de autores italianos. En dichas relaciones, el relato de los usos y costumbres, formas de vida, prácticas devocionales, creencias, modas locales y placeres culinarios adquiere una dimensión que permite la reconstrucción de un mapa de indumentarias, sabores y supersticiones todavía no suficientemente explorado por los exper-

\footnotetext{
Ambrosio Ignacio de Spínola y Guzmán (Madrid, 1632 - Sevilla, 1684) fue arzobispo de Compostela muy brevemente, entre 1668 y 1669, coincidiendo su pontificado casi con el lapso del viaje de Cosimo de' Medici por la península. Era nieto del gran capitán de los tercios de Flandes, Ambrosio Spinola Doria (Génova, 1569 -Castelnuovo Scrivia, 1630), I duque de Sesto, I marqués de los Balbases y Grande de España, inmortalizado por Velázquez recogiendo las llaves de la ciudad holandesa de Breda, en señal de rendición. Por lo tanto, el arzobispo de Santiago de Compostela y el príncipe de Toscana compartían origen italiano y sus respectivas familias habían estado relacionadas. Probablemente ellos ni lo recordasen, o no fuese un tema edificante sobre el que $\mathrm{Su}$ Alteza Serenísima y Su Excelencia Reverendísima tuviesen a bien departir, pero lo cierto es que Simonetta Vespucci (1454-1476), hija de Cattolica Spinola, fue el gran amor de Giuliano de' Medici (Florencia, 1453-1478), hermano de Lorenzo el Magnífico, y la musa de media Florencia, como lo demuestran los cuadros de Sandro Botticelli, que la retrató en El nacimiento de Venus.
} 
tos en estos ámbitos disciplinares, ya que, en su mayoría, los manuscritos citados continúan inéditos casi tres siglos y medio después.

b. La dimensión intercultural que ofrecen estos testimonios permite establecer dimensiones comparatistas entre las culturas itálicas e ibéricas, ofreciendo en el caso ibérico un detallado recorrido por la diversidad cultural que España y Portugal albergan dentro de sus fronteras.

c. Desde el punto de vista filológico, dicho trabajo comparatista permite identificar variaciones léxicas, algunas de ellas influidas por el contacto con las lenguas locales, que los cronistas introducen en su lengua original a medida que se familiarizan con la terminología utilizada en la Península ibérica.

\section{Referencias bibliográficas}

Astete, Gaspar de (1597): Libro primero y segundo del gobierno de la familia y estado del matrimonio, Madrid, [s.n.].

Casas Gaspar, Enrique (1947): Costumbres españolas de nacimiento, noviazgo, casamiento y muerte, Madrid, Escelicer.

Corsini, Filippo (1669): Viaggi d'Alemagna, Paesi Bassi del 1667 e di Spagna, Francia, Inghiterra e Olanda dal 1668 e 1669, fatti dal Ser.mo Principe Cosimo di Toscana di poi Gran Duca Terzo di quel nome, scritti dal Marchese Filippo Corsini coppiere di S.A.R. e figliuolo del Marchese Bartolomeo Corsini, Archivio di Stato di Firenze, Mediceo del Principato, 6387; Miscellanea medicea, 838.

Florez Asensio, María Asunción (2010): «El Marqués de Liche: Alcaide del Buen Retiro y "Superintendente" de los festejos reales», Anales de Historia del Arte, 20, pp. 145-182.

González Fernández, Isabel (2004): «Compostela en las crónicas del segundo viaje por Europa de Cosme III de Médicis», en Xosé A. Neira Cruz (ed.), El viaje a Compostela de Cosme III de Médicis. Santiago de Compostela, Santiago de Compostela, Xunta de Galicia. Consellería de Cultura, Comunicación Social e Turismo. S.A. de Xestión do Plan Xacobeo, pp. 231-249.

Gornia, Giovan Battista (1669): Viaggio fatto dal Serenissimo Principe Cosimo Terzo di Toscana per la Spagna, Inghilterra, Francia et altri luoghi negli anni 1668 e 1669, scritto dal Dottor Giov. Batt. ${ }^{a}$ Gornia Bolognese, quale in qualità di Medico viaggiò con S.A.R., Archivio di Stato di Firenze, Mediceo del Principato, 6389; Miscellanea medicea, 839.

Marchisio, Cristina (2004): «Siguiendo la senda de los búcaros. Cosme III de Toscana en España y Portugal (1668-1669)», en Xosé A. Neira Cruz (ed.), El viaje a Compostela de Cosme III de Médicis, Santiago de Compostela, Xunta de Galicia. Consellería de Cultura, Comunicación Social e Turismo. S.A. de Xestión do Plan Xacobeo, pp. 287-307.

Neira Cruz, Xosé A. (ed.) (2004): El viaje a Compostela de Cosme III de Médicis. Santiago de Compostela, Xunta de Galicia. Consellería de Cultura, Comunicación Social e Turismo. S.A. de Xestión do Plan Xacobeo.

Neira Cruz, Xosé A. (2015): Los albores de la crónica periodística: las crónicas del viaje por España y Portugal de Cosimo III de'Medici, Tesis doctoral defendida en el Departamento de Filología Francesa e Italiana de la Universidad de Santiago el 9 de diciembre de 2015.

Neira Cruz, Xosé A. (2016): «En los corrales de comedias. Una propuesta de identificación de las obras de teatro que Cosimo dei Medici presenció durante su estancia en Madrid», en Carmen Blanco Valdés, Ana María Domínguez Ferro (eds.), Madonna á 'n sé vertute 
con valore: Estudio en homenaje a Isabel González, Córdoba / Santiago de Compostela, Universidad de Córdoba / Universidad de Santiago de Compostela, pp. 215-225.

Palomero Páramo, Jesús (2004): «Andalucia. Toros, caballos y música para un príncipe florentino», en Xosé A. Neira Cruz (ed.), El viaje a Compostela de Cosme III de Médicis, Santiago de Compostela, Xunta de Galicia. Consellería de Cultura, Comunicación Social e Turismo. S.A. de Xestión do Plan Xacobeo, pp. 437-483.

Salort Pons, Salvador (2004): «Relaciones artísticas entre las cortes de Madrid y Florencia en tiempos de Cosme III», en Xosé A. Neira Cruz (ed.), El viaje a Compostela de Cosme III de Médicis, Santiago de Compostela, Xunta de Galicia. Consellería de Cultura, Comunicación Social e Turismo. S.A. de Xestión do Plan Xacobeo, pp. 347-361.

Sánchez Rivero Ángel / Mariutti de Sánchez Rivero Angela (1933): Viaje de Cosme de Médicis por España y Portugal (1668-1669), Madrid, Junta para la Ampliación de Estudios e Investigaciones Científicas, Centro de Estudios Históricos / Imprenta Herederos de Ribadeneyra. 